Ciência eNatura, Santa Maria, v. 37 Part 1 2015, p. 190-193

\title{
Design a mechanism Like flapping hummingbird wings
}

\author{
Mohamad Hasan Javareshkian ${ }^{1}$, Mehdi Behnam Vashani² \\ ${ }^{1}$ Associate Professor, Department of Mechanical Engineering, Ferdowsi University of Mashhad, Mashhad,Iran \\ ${ }^{2}$ Msc Student, Department of Mechanical Engineering, Ferdowsi University of Mashhad, Mashhad, Iran
}

\begin{abstract}
Hummingbirds have been considered because they have high maneuverability. Making an example of this type of bird needs to observe the kinematic and aerodynamic issues of the bird's flight. In this article the locus of attack angle and instantaneous elevation is studied to be used for constructing a sample of birds. At first, the charts offered in the article of bird's flight was digitized and by using MATLAB software was fitted to the shape of a sinusoidal equation to be comparable with designed mechanisms. The designed mechanisms have three variables that by using EES software the motion equation has been approached. Then by the Lagrange Interpolation the effect of each variable in the equation of motion mechanism obtained. Finally by using EES software these equation is solved and the amount of the variables was calculated.
\end{abstract}

Keywords: Aerodynamics, Flapping Wing, Lagrange Interpolation, Hummingbird, Mechanism. 


\section{Introduction}

$\mathrm{A}$ fter the man understood flight, began to construct and design flying vehicles. After years, by scientists and engineer's effort in 1903 Wright brothers' first successful flight took place and man shortly could feel the taste of flight. Now, after more than 10 years, High proportion of transport done by flying vehicles and every day added to the loads and distance flight. Nowadays, micro air vehicles are used for rescuing, entering to dangerous places and security situations and for this reasons, the controllable micro air vehicles have been improved a lot in last years.

Micro air vehicles have dimension smaller than $15 \mathrm{~cm}$, speed $10 \mathrm{~m} / \mathrm{s}$, Reynolds number based on main chord less than 150000.[1] Because of the high weight of the battery and control devices with today's technology, the long flights are not possible. [2]

recently the idea for a new kind of flying vehicles, means that making flying vehicles the same as dimension and amount of the real birds and insects and by imitating from their flight system, formed.

This kind of flying vehicle instead of using a propeller by flapping it produces thrust. This kind of flying vehicle can hover.[3] All flying creatures of nature fly by flapping their wings and according to researches of Bionics and Aerodynamics, flapping-wing flight is proved to embody more advantages than both fixed-wing flight and rotary-wing flight.[7]

These advantages conclude higher aerodynamic efficiency, bigger load, more convenient landing, higher altitude and Moderate speed. Generally speaking, this kind of product is applied in military reconnaissance field as a surveillance equipment of a single soldier. Therefore, it can be equipped with surveillance equipment with apparent application value.

\subsection{From the perspective of biology:}

The birds are more effective than the other animals in man life.[8] Birds, flying vertebrates, have wings and the most important feature is having feather. There are more than 9700 spices bird around the world.[9]

The biggest alive flightless bird, the African ostrich, height $2.5 \mathrm{~m}$, weight $150 \mathrm{~kg}$. Within the flying birds, the biggest one is American vulture, with wingspan $3 \mathrm{~m}$.[8] Birds which weigh more than $15-17 \mathrm{~kg}$ can't flap fast to fly horizontally. Smaller birds have the advantages of being able to use different flapping frequencies, but for animals with a weight of about $1 \mathrm{~g}$ there is another upper limitation. Their muscles need time to reset the contractile mechanism after each contraction (Norberg 1990).

For insects with wing-beat frequencies up to $400 \mathrm{~Hz}$, this problem is solved with special fibrillar muscles[10] capable of contracting and resetting at very high frequencies.[11]

\subsection{From the perspective of aerodynamic:}

The features of micro air vehicles:

1) Low Reynolds number because of the bad aerodynamic condition for controlling.

2) Small physical dimensions because of issues such as structural strength, reduced failure speed and low inertia usually enlarge in tests.

3) Because of the low inertia and low speed this birds are sensitive to flow disturbances.

Hovering hummingbird [1-7] Reynolds number is about 3000.[12]

Flapping of birds during flight has 4 general pattern: 1 . flutter 2 . reverse 3 . swing 4 . folding

Flutter means change the angle of the wings around the move direction.

Reverse means rotation around span wise.

Swing means change the vertical angle of the wing of body.

Folding means bending and flattening of the wing.

In bigger birds reverse and swing are negligible.[7]

Flight modes of birds and insects are hovering, forward flight, and rotation.

During flying from one flight mode to the other one, they quickly change their feature as angle of attack.

Birds produced thrust just during flapping. 
In hummingbirds and insects flight lift is produced during the entire wing stroke. Refrens [12] used DPIV toob serve the wake around hovering hummingbirds. They observed force asymmetry between the upstroke and the down stroke. Specifically, $75 \%$ of the lift is generated during the down stroke and $25 \%$ during the upstroke. They reported inversion of the cambered wings during the upstroke, as well as evidence of LEVs, created during the down stroke. As suggested by the Reynolds number, a hummingbird's aerodynamics regime overlaps that of larger insects.[10]

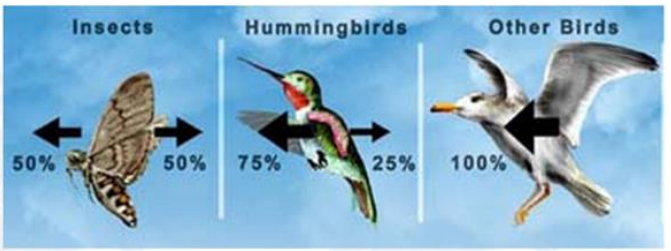

Figure 1: Difference between larger birds, insects and hummingbird's flight[13]

\subsection{From the perspective of mechanics:}

Hummingbird flapping equation in the general case can be shown as follows:
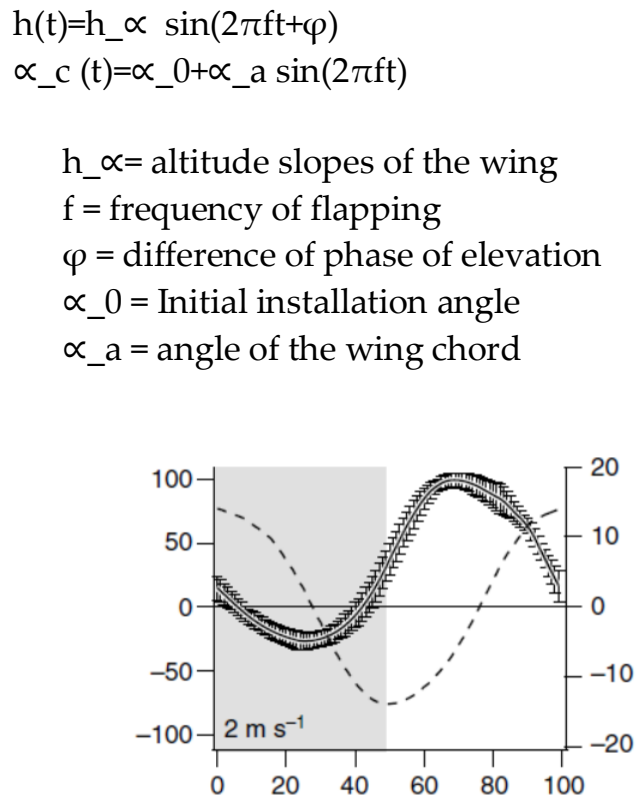

Figure 2: Locus and angle of wrist cord at speed $2 \mathrm{~m} / \mathrm{s}$ [19]

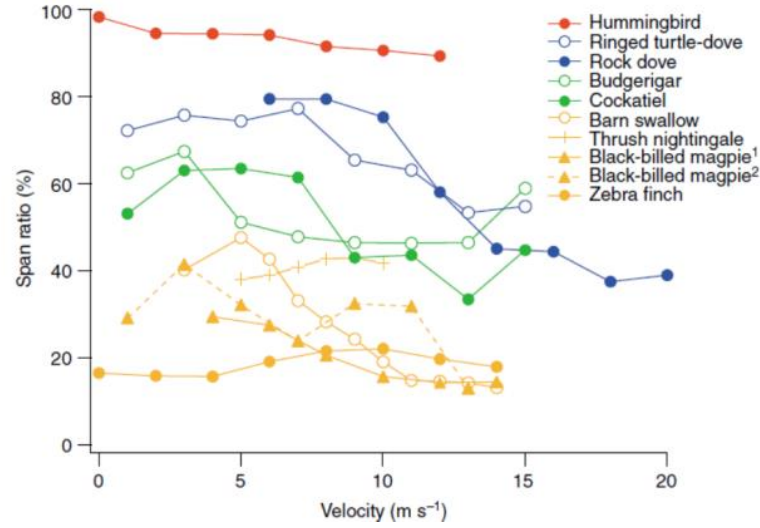

Figure 3: Coefficient of variation wingspan in a cycle of flapping for different birds.[2]

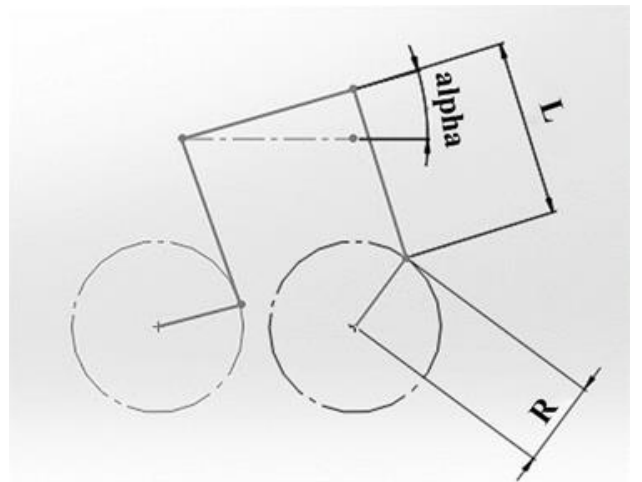

Figure 4: designed flapper mechanisms similar to hummingbird.

\subsection{Polynomial interpolation of Lagrange}

The nodes, Are the known points of the function $\mathrm{F}$. According to the Weierstrass approximation theorem $\mathrm{P}$, polynomial interpolation of function $\mathrm{F}$, can be found by having node information, with the error $\varepsilon$. The function $\mathrm{P}$ in one or more points matches on the function $\mathrm{F}$. It can be proved that the polynomial function $\mathrm{P}$ is unique.

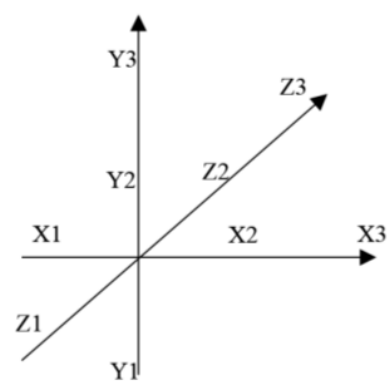

Figure 5: Three-dimensional Lagrange Interpolation nodes

In the late 19th century need to solve multidimensional interpolation functions led to multivariate polynomial model Lagrange be 
Presented. This polynomial type, proposes a surface or a space includes the nodes.[15-118] In this case Lagrange basis polynomials is as below:

$$
L_{i, j, k}(x, y, z)=L_{i} \times L_{j} \times L_{k}
$$

And polynomial interpolation of Lagrange will be as follows:

$$
P(x)=\sum_{i=0}^{n} \sum_{i=0}^{n} \sum_{i=0}^{n} L_{i, j, k}(x, y, z) F\left(x_{i}, y_{j}, z_{k}\right)
$$

Lagrange interpolation function approximates non-complex and non-monotonic equations with good accuracy.[14] Thus first the equation was checked. For more than 1800 nodes, the equation was univocal. Threedimensional Lagrange interpolation function was written in MATLAB as a code. The code calculates three variables crank radius, arm and the phase difference of leading edge and trailing edge.

\section{Conclusion}

In this paper aerodynamics, flight mechanics and kinematics of flapping wings of birds and insects are studied, as well as activities performed in this field. Conducted research concluded that the governing equations of steady aerodynamic to calculate lift and thrust forces in flapping bird's wing is Insufficient. In the field of control and stability of MAVs, researches and studies are needed.

\section{References}

Y. Shyy W, Computational Investigation of Unsteady Low-Reynolds Number Aerodynamics for Micro Air Vehicles, Michigan Univ., Ann Arbor. Dept. of Aerospace Engineering, pp. 2007.

O. Breitenstein, Development of a Flapping Wing Mechanism, Thesis, Autonomous Systems Lab, Swiss Federal Institute of Technology (ETH) Zurich, Autonomous Systems Lab, 2009.

W. Shyy, M. Berg, D. Ljungqvist, Flapping and flexible wings for biological and micro air vehicles, Progress in Aerospace Sciences, Vol. 35, No. 5, pp. 455-505, 7//, 1999.

J. Alderfer, Complete Birds of North America: national geographic, 2006.

G. A. Harrison C, Birds of The World: Dorling Kindersley, 2000.

P. Christiansen, Birds: Amber books, 2009.

C. J. Liang B, ZhangL, Research on Birds Flapping-Wing Bionic Mechanism, Mechanical Engineering Research, Vol. 1, No. 1, 2011.

L. Y. shyy W, Tang J, Liu H, Viieru D, Liu H, Aerodynamics of Low Reynolds Number Flyers, 2008.

S. J. Kirkpatrick, Scale effects on the stresses and safety factors in the wing bones of birds and bats, The Journal of Experimental Biology, Vol. 190, No. 1, pp. 195-215, May 1, 1994, 1994.

T. W. Warrick D R, Powers D R, Aerodynamics of the hovering hummingbird, Nature, Vol. 435, pp. 1094-1097, 23 June 2005, 2005.

http://people.eku.edu/, Accessed.

http://www.iust.ac.ir/files/math/numerical_analy sis_1.pdf

W. C. Won Young Yang, Tae-Sang Chung, John Morris, APPLIED NUMERICAL METHODS USING MATLAB, Canada: A JOHN WILEY \& SONS, INC, 2005.

D. Levy, Introduction to Numerical Analysis, Thesis, Department of Mathematics and Center for Scientific Computation and Mathematical Modeling (CSCAMM) University of Maryland, 2010.

R. J. T D Patzek, An introduction to computer programing for engineering and scientists, Third ed.: University of California, 2006.

G. Muntingh, Topics in Polynomial Interpolation Theory, Philosophie Doctor Thesis, Centre of Mathematics for Applications, Oslo, 2010.

Bret W. Tobalske, D.R.W., Christopher J. Clark, Donald R. Powers Tyson L. Hedrick, Gabriel A. Hyder ,ndrew A. Biewener, "Threedimensional kinematics of hummingbird flight", The Journal of Experimental Biology, Vol., 2368-2382, 2007. 